DOI: $10.1590 / 1089-6891 v 18 \mathrm{e}-16840$

RECURSOS PESQUEIROS E ENGENHARIA DE PESCA

\title{
DENSIDADE PLANCTÔNICA DO POLICULTIVO DE Litopenaeus vannamei E Oreochromis niloticus
}

\section{PLANKTON DENSITY IN Litopenaeus vannamei AND Oreochromis niloticus POL YCULTURE}

\author{
Luis Otavio Brito ${ }^{1 *}$ \\ Bruno Rodrigo Simão ${ }^{2}$ \\ João Batista Pereira Neto ${ }^{3}$ \\ Gabriela Cemirames ${ }^{4}$ \\ Celicina Maria da Silveira Borges de Azevedo ${ }^{5}$ \\ ${ }_{1}^{1}$ Universidade Federal Rural de Pernambuco, Recife, PE, Brasil
${ }^{2}$ Universidade Federal Rural do Semi-Árido, Mossoró, RN, Brasil
${ }^{3}$ InVivo Nutrição e Saúde Animal Ltda., Brasil
${ }^{4}$ Universidade Federal Rural do Semi-Árido, Mossoró, RN, Brasil
${ }^{5}$ Universidade Federal Rural do Semi-Árido, Mossoró, RN, Brasil
${ }^{*}$ Autor para correspondência - engpescalo@hotmail.com
}

\section{Resumo}

Um experimento foi realizado durante 95 dias para avaliar as densidades planctônicas do policultivo do camarão branco Litopenaeus vannamei e da tilápia do Nilo Oreochromis niloticus. O delineamento experimental foi inteiramente casualizado com seis tratamentos e quatro repetições cada. Os tratamentos foram: monocultivo com 10 camarões $\mathrm{m}^{2}$ (10S:0T); policultivo com 10 camarões e 0,5 tilápia $\mathrm{m}^{2}$ (10S:0.5T); policultivo com 10 camarões e 1 tilapia $\mathrm{m}^{2}(10 \mathrm{~S}: 1 \mathrm{~T})$; monocultivo com 2 tilápias $\mathrm{m}^{2}$ (2T:0S); policultivo com 2 tilápias e 2,5 camarões $\mathrm{m}^{2}(2 \mathrm{~T}: 2,5 \mathrm{~S})$; e policultivo com 2 tilápias e 5 camarões $\mathrm{m}^{2}$ (2T:5S). Não foi realizada troca de água e nem fornecimento de aeração artificial durante o período experimental, apenas utilizou-se água para completar o volume perdido pela evaporação. O fitoplâncton variou de 78.981 a 303.260 cél. $\mathrm{mL}^{-1} \mathrm{e}$ o zooplâncton variou de 470 a 1.421 org. $\mathrm{L}^{-1}$. Os grupos mais frequentes foram Bacillariophyta (fitoplâncton) e Rotífera (zooplâncton). Pode-se concluir que em sistema de policultivo do camarão branco (Litopenaeus vannamei) e a tilápia do Nilo (Oreochromis niloticus) não ocorrem modificações significativas nas densidades do fitoplâncton e zooplâncton em decorrência da alteração das densidades de estocagem do cultivo.

Palavras-chave: camarão; fitoplâncton; tilapia; zooplâncton.

\begin{abstract}
An experiment was conducted during 95 days to evaluate plankton densities in polyculture of Pacific white shrimp Litopenaeus vannamei and Nile tilapia Oreochromis niloticus. The experiment design was randomized with three treatments and four replicates each. The treatments were as follows: monoculture with 10 shrimps $\mathrm{m}^{2}(10 \mathrm{~S}: 0 \mathrm{~F})$; polyculture with 10 shrimps and 0.5 fish $^{2}$
\end{abstract}


(10S:0.5F); polyculture with 10 shrimps and 1 fish $\mathrm{m}^{2}(10 \mathrm{~S}: 1 \mathrm{~F})$; monoculture with 2 fishes $\mathrm{m}^{2}$ (2F:0S); polyculture with 2 fishes and 2.5 shrimps $\mathrm{m}^{2}$ (2F:2.5S); and polyculture with 2 fishes and 5 shrimps $\mathrm{m}^{2}(2 \mathrm{~F}: 5 \mathrm{~S})$. Neither water exchange nor aeration was provided during the experimental period, only water was used to complete the volume lost by evaporation. The phytoplankton ranged from 78,981 to 303,260 cells. $\mathrm{mL}^{-1}$ and the zooplankton ranged from 470 to 1,421 org. $\mathrm{L}^{-1}$. The most frequent groups were Bacillariophyta (phytoplankton) and Rotifera (zooplankton). In conclusion, in the polyculture system of Pacific white shrimp Litopenaeus vannamei and Nile tilapia Oreochromis niloticus there are no significant changes in the densities of phytoplankton and zooplankton due to the alteration in the culture stocking density.

Keywords: phytoplankton; shrimp; tilapia; zooplankton.

Recebido em: 11 janeiro de 2012

Aceito em: 28 abril 2017

\section{Introdução}

Os organismos fitoplanctônicos (ex. Bacillariophyta e Chlorophyta), zooplanctônicos (ex. Rotífera, Cladocera e Copépoda) e fitobentônico (ex. Bacillariophyta Pennales e Centrales) são componentes da biota natural disponíveis em viveiros de cultivo de organismos aquáticos ${ }^{(1-3)}$. Esta biota natural pode ser a chave para o sucesso do cultivo, pois os nutrientes e resíduos das rações são em grande parte reciclados, melhorando a qualidade do ambiente ${ }^{(4)}$.

Além da importância para a qualidade da água, a biota natural serve de alimento natural para os camarões $^{(5,6)}$ e tilápias ${ }^{(7,8)}$. O manejo adequado desta biota natural pode contribuir significativamente na melhora da viabilidade econômica dos cultivos de espécies aquícolas ${ }^{(9)}$. Entretanto, a abundância do plâncton é dependente da dinâmica de nutrientes, que é influenciada pelas práticas de manejo e pelas densidades de estocagem ${ }^{(10)}$.

Camarões da espécie Litopenaeus vannamei são predadores eficientes do plâncton, mas a eficiência de predação está relacionada com o tamanho dos camarões ${ }^{(4)}$. Na fase de náuplios, alimentam-se da reserva de alimento depositada nos ovos, a partir do estágio larval de zoéa e misis precisam de um alimento vivo para satisfazer as suas necessidades nutricionais e energéticas, enquanto no estágio de pós-larva tendem a se tornar gradualmente onívoros ${ }^{(11)}$. Já a tilápia Oreochromis niloticus em viveiros com arraçoamento apresenta no trato digestório organismos macrobênticos (Chironomidae); macrófitas (Salvinia auriculata); zooplâncton (Cladocera Moina micrura e Daphnia sp.); e algas (Scenedesmus opoliensis, Coelastrum pseudomicroporum, C. astroideum e Crucigenia tetrapedia $)^{(12)}$.

A adoção de um sistema de policultivo de tilápias com camarões tem se expandido entre os produtores em muitos países do mundo como uma alternativa para aumentar a sustentabilidade dos cultivos. Essa estratégia de produção, na qual se combinam duas ou mais espécies complementares, pode proporcionar aumento na produtividade em função de ajuste na estrutura da cadeia trófica, que é rearranjada para melhor utilização do alimento natural, reduzindo a demanda de alimento $\operatorname{artificial}^{(13-15)}$.

As tilápias podem ser eficazes no controle biológico de algas ${ }^{(16)}$ e na reciclagem de resíduos ${ }^{(14)}$; entretanto, depende da sua densidade de estocagem. Isto se torna importante em ambientes de 
cultivo de camarões, pois ocorre um aumento significativo da concentração de nutrientes ${ }^{(17-19)}$ e na densidade de algas ${ }^{(20-22)}$ durante o ciclo de cultivo.

Neste sentido, o objetivo deste trabalho foi avaliar a composição do plâncton no policultivo de camarão branco (L. vannamei) e tilápia do Nilo (O. niloticus).

\section{Material e métodos}

O experimento foi realizado no Setor de Aquicultura da Universidade Federal Rural do Semi-Árido - UFERSA, no Município de Mossoró/RN (511' S de Latitude, 37²0' W de Longitude), durante 95 dias, utilizando-se delineamento inteiramente casualisado com seis tratamentos e quatro repetições em cada. Os tratamentos foram os seguintes: monocultivo com 10 camarões $\mathrm{m}^{2}$ (10S:0T); policultivo com 10 camarões e 0,5 tilápia $\mathrm{m}^{2}$ (10S:0.5T); policultivo com 10 camarões e 1 tilápia $\mathrm{m}^{2}$ (10S:1T); monocultivo com 2 tilápias $\mathrm{m}^{2}$ (2T:0S); policultivo com 2 tilápias e 2,5 camarões $\mathrm{m}^{2}$ (2T:2,5S); e policultivo com 2 tilápias e 5 camarões $\mathrm{m}^{2}$ (2T:5S).

As pós-larvas de camarões L. vannamei $\left(\mathrm{PL}_{12}\right)$ oriundas de larvicultura comercial (Compescal Larvicultura Ltda., Aracati, CE) e os alevinos de tilápia do Nilo (O. niloticus) de larvicultura governamental (Departamento Nacional de Obras Contra as Secas, DNOCS, Caicó, RN) foram aclimatados para 4 g.L $\mathrm{L}^{-1}$ de salinidade em tanques berçários por 30 dias. Durante a aclimatação, os camarões foram alimentados com biomassa de Artemia (2g/10.000 PL) e ração comercial $(0,4$ a $1,0 \mathrm{~mm})$ com $40 \%$ proteína bruta $(\mathrm{PB})(25 \text { a } 10 \% \text { da biomassa })^{(23)}$ e as tilápias com ração comercial $(2 \mathrm{~mm})$ com 32\% PB (12 a 10\% da biomassa) três vezes por dia $(08 \mathrm{~h}, 12 \mathrm{~h} \text { e } 16 \mathrm{~h})^{(24)}$.

Após o período de aclimatação, os camarões $(0,34 \pm 0,01 \mathrm{~g})$ e tilápias $(8,22 \pm 0,53 \mathrm{~g})$ foram estocados em tanques de alvenaria com área de $15 \mathrm{~m}^{2}$, profundidade de $1,2 \mathrm{~m}$ e com uma camada de cinco $\mathrm{cm}$ de solo, preenchidos com água de poço tubular, com salinidade de $4 \mathrm{~g} . \mathrm{L}^{-1}$. Nos tratamentos (10S:0T), (10S:0.5T) e (10S:1T) os camarões foram alimentados com (2 a 2,5 mm) de $30 \%$ PB, oferecida em bandejas, com base na biomassa (5,5 a 2,5\%) do camarão em uma frequência de três vezes ao dia $(8 \mathrm{~h}, 12 \mathrm{~h} \text { e } 16 \mathrm{~h})^{(24)}$, enquanto para as tilápias não foi ofertado nenhum tipo de alimento. Nos tratamentos $(2 \mathrm{~T}: 0 \mathrm{~S}) ;(2 \mathrm{~T}: 2,5 \mathrm{~S})$ e $(2 \mathrm{~T}: 5 \mathrm{~S})$ as tilápias foram alimentadas com ração comercial $(4 \mathrm{~mm})$ de $32 \%$ PB oferecida a voleio, com base na biomassa (10 a $2 \%$ ) dos peixes em uma frequência de três vezes ao dia $(8 \mathrm{~h}, 12 \mathrm{~h} \text { e } 16 \mathrm{~h})^{(23)}$, enquanto para os camarões não foi ofertado nenhum tipo de alimento.

Durante o período de cultivo não houve renovação de água e nem aeração artificial, apenas abastecimento de água para suprir a evaporação. Uma semana antes de os animais serem estocados nas unidades experimentais, os tanques foram fertilizados inicialmente com ureia e superfosfato numa proporção de $20 \mathrm{Kg}$ de nitrogênio e $10 \mathrm{Kg}$ de fósforo por hectare ${ }^{(24)}$. Nitrato de sódio foi utilizado durante o período de cultivo para estimular a produção do alimento natural (Tabela 1).

As amostras semanais de plâncton foram coletadas verticalmente em garrafas plásticas com volume de $500 \mathrm{~mL}$ na direção fundo-superfície, de modo que percorreu toda coluna de água dos tanques. Com intuito de tornar as amostras mais representativas, foi realizada a filtragem do plâncton em 
mini-rede de $15 \mu \mathrm{m}$ (fitoplâncton) ${ }^{(25)}$ e $50 \mu \mathrm{m}$ (zooplâncton) ${ }^{(26)}$. O material foi concentrado em um volume de $500 \mathrm{~mL}$ para $10 \mathrm{~mL}$, representando assim uma amostra 50 vezes mais concentrada. $\mathrm{O}$ material recém-coletado foi imediatamente fixado com formol a 4\% e tamponado com bórax $(1 \%)^{(25)}$.

Tabela 1. Fertilização dos tanques com nitrato de sódio durante o período experimental

\begin{tabular}{lcccc}
\hline \multirow{2}{*}{ Fertilização } & & \multicolumn{2}{c}{ Quantidade $\mathbf{( k g \cdot h \mathbf { H } ^ { - 1 } )}$} \\
Semana & 30 & 20 & 10 & 0 \\
& $2-4$ & $5-7$ & $8-10$ & $11-13$ \\
\hline
\end{tabular}

Para análise quantitativa do plâncton, foi utilizado microscópio óptico binocular (OLYMPUS CH30) com aumento de 800 vezes de imagem total, além de consultas a referências bibliográficas $^{(27-29)}$.

Diariamente, foram mensuradas as variáveis da água, temperatura e oxigênio dissolvido (YSI 550a Yellow Springs, Ohio, EUA). O pH (pH-1700, Instrutherm Instrumentos de Medição Ltda., São Paulo, SP, Brasil) e salinidade (refratômetro portátil, 211 Briobrix Equipar Ltda., Curitiba, PR, Brazil) foram mensurados uma vez por semana.

Para as variáveis de qualidade da água previamente constatou-se a homogeneidade das variâncias (Cochran) e normalidade dos dados (Shapiro-Wilk) e ANOVA de medidas repetidas foi aplicada em seguida. Os dados de densidade do fitoplâncton e zooplâncton foram previamente submetidos à análise de homogeneidade das variâncias (Cochran) e normalidade dos dados (Shapiro-Wilk) e em seguida, devido aos dados não serem paramétricos, foi utilizado o teste de Kruskal-Wallis (teste H). Todas as análises foram realizadas com auxílio do software ASSISTAT versão 7.6.

\section{Resultados}

Os valores médios observados de temperatura $\left({ }^{\circ} \mathrm{C}\right)$ e oxigênio dissolvido $\left(\mathrm{mg} . \mathrm{L}^{-1}\right)$ pela manhã e à tarde, $\mathrm{pH}$ e salinidade não apresentaram diferença significativa entre os tratamentos (Tabela 2).

As densidades do fitoplâncton estão sumarizadas na Tabela 3. O grupo Bacillariophya foi o mais abundante, seguido de Cyanophyta, Chlorophyta e Pyrrophyta. As densidades médias de microalgas totais (cél. $\mathrm{mL}^{-1}$ ) variaram entre 78.981 a 303.260 , Bacillariophyta (cél. $\mathrm{mL}^{1}$ ) entre 55.406 a 269.360, Cyanophyta (cél. $\mathrm{mL}^{-1}$ ) entre 9.856 a 23.494, Chlorophyta (cél. mL ${ }^{1}$ ) entre 2.025 a 8.868 e Pyrrophyta (cél. $\mathrm{mL}^{-1}$ ) entre 3.900 a 4.931 , não apresentando diferença significativa entre os tratamentos (Tabela 3).

As densidades do zooplâncton estão sumarizadas na Tabela 4. O grupo Rotífera foi o mais abundante, seguido de Cladocera e Copépoda. As densidades médias do zooplâncton total (org. $\mathrm{L}^{-1}$ ) variaram entre 470 a 1.421 , Rotífera (org. $\mathrm{L}^{-1}$ ) entre 467 a 1.399 , Cladocera (org. $\mathrm{L}^{-1}$ ) entre 0,48 a 3,80, Copépoda (org. $\mathrm{L}^{-1}$ ) entre 0,04 a 1,95 e outros grupos (org. $\mathrm{L}^{-1}$ ) entre 2,36 a 7,39, não apresentando diferença significativa entre os tratamentos (Tabela 4). 
Tabela 2. Variáveis da qualidade da água em tanques de policultivo de camarões e tilápias

\begin{tabular}{|c|c|c|c|c|c|c|}
\hline \multirow[t]{2}{*}{ Tratamentos } & \multicolumn{6}{|c|}{ Variáveis } \\
\hline & $\begin{array}{c}\text { OD } \\
\left(\mathrm{mg} \cdot \mathbf{L}^{-1}\right) \\
\text { (manhã) }\end{array}$ & $\begin{array}{c}\text { OD } \\
\left(\text { mg.L }^{-1}\right) \\
\text { (tarde) }\end{array}$ & $\begin{array}{c}\text { Temp } \\
\left({ }^{\circ} \mathrm{C}\right) \\
\text { (manhã) }\end{array}$ & $\begin{array}{c}\text { Temp } \\
\left({ }^{\circ} \mathrm{C}\right) \\
\text { (tarde) }\end{array}$ & pH & $\begin{array}{l}\text { Salinidade } \\
\left(\text { g. } \mathrm{L}^{-1}\right)\end{array}$ \\
\hline $10 \mathrm{C}: 0 \mathrm{~T}$ & $8,01 \pm 0,37$ & $12,26 \pm 0,77$ & $30,22 \pm 0,21$ & $32,02 \pm 0,42$ & $7,69 \pm 0,05$ & $2,68 \pm 0,05$ \\
\hline $10 \mathrm{C}: 0,5 \mathrm{~T}$ & $7,66 \pm 0,42$ & $12,11 \pm 0,23$ & $30,12 \pm 0,27$ & $32,05 \pm 0,39$ & $7,70 \pm 0,07$ & $2,76 \pm 0,17$ \\
\hline $10 \mathrm{C}: 1 \mathrm{~T}$ & $7,48 \pm 0,57$ & $11,31 \pm 1,05$ & $30,26 \pm 0,27$ & $32,21 \pm 0,31$ & $7,63 \pm 0,11$ & $2,72 \pm 0,09$ \\
\hline $2 \mathrm{~T}: 0 \mathrm{C}$ & $5,32 \pm 0,85$ & $11,11 \pm 0,91$ & $30,31 \pm 0,04$ & $32,31 \pm 0,11$ & $7,50 \pm 0,06$ & $2,81 \pm 0,13$ \\
\hline $2 \mathrm{~T}: 2,5 \mathrm{C}$ & $5,46 \pm 0,26$ & $11,76 \pm 0,37$ & $30,29 \pm 0,11$ & $32,20 \pm 0,12$ & $7,54 \pm 0,07$ & $2,80 \pm 0,18$ \\
\hline $2 \mathrm{~T}: 5 \mathrm{C}$ & $5,41 \pm 0,31$ & $11,52 \pm 0,39$ & $30,17 \pm 0,24$ & $31,95 \pm 0,23$ & $7,55 \pm 0,02$ & $2,74 \pm 0,28$ \\
\hline
\end{tabular}

Tabela 3. Composição do fitoplâncton em tanques de policultivo de camarões e tilápias

\begin{tabular}{lccccccc}
\hline \multicolumn{1}{c}{ Grupos } & \multicolumn{7}{c}{ Tratamentos* } \\
\hline \multicolumn{1}{c}{ cel.mL $^{-1}$ ) } & 10C:0T & 10C:0,5T & 10C:1T & 2T:0C & 2T:2,5C & 2T:5C & \\
\hline Fitoplâncton total & 84.637 & 81.875 & 78.981 & 303.260 & 134.311 & 126.512 & 5.418 \\
Bacillariophyta & 57.165 & 56.015 & 55.406 & 269.370 & 109.391 & 109.103 & 1.487 \\
Chlorophyta & 4.338 & 4.038 & 2.025 & 8.868 & 4.893 & 2.783 & 2.035 \\
Cyanophyta & 23.494 & 16.763 & 13.697 & 20.546 & 16.127 & 9.856 & 7.679 \\
Pyrrophyta & 4.931 & 3.910 & 3.713 & 4.770 & 4.476 & 3.900 & 2.298 \\
\hline
\end{tabular}

*Valores são medias; **Teste de Kruskal-Wallis (teste $\mathrm{H}=11.070$ )

Tabela 4. Composição do zooplâncton em tanques de policultivo de camarões e tilápias

\begin{tabular}{|c|c|c|c|c|c|c|c|}
\hline \multirow{2}{*}{$\begin{array}{c}\text { Grupos } \\
\text { (org.L-1) }\end{array}$} & \multicolumn{6}{|c|}{ Tratamentos* } & \multirow[t]{2}{*}{$\mathbf{H}^{* *}$} \\
\hline & 10C:0T & 10C:0,5T & 10C:1T & 2T:0C & $2 \mathrm{~T}: 2,5 \mathrm{C}$ & 2T:5C & \\
\hline Zooplâncton total & 757 & 682 & 470 & 1.407 & 1.168 & 734 & 1.750 \\
\hline Rotifera & 748 & 677 & 467 & 1.399 & 1.159 & 728 & 1.519 \\
\hline Cladocera & 3,80 & 1,88 & 0,48 & 3,80 & 1,38 & 1,08 & 4.985 \\
\hline Copépoda & 1,80 & 1,07 & 0,21 & 1,95 & 0,12 & 0,04 & 5.812 \\
\hline Outros & 4,08 & 3,08 & 2,36 & 7,39 & 4,36 & 3,02 & 1.421 \\
\hline
\end{tabular}

*Valores são medias; **Teste de Kruskal-Wallis (teste $\mathrm{H}=11.070$ )

\section{Discussão}

As variáveis de qualidade de água estiveram dentro da faixa de conforto para as espécies estudadas $^{(30,31)}$, não ocorrendo variações bruscas que, segundo Preston et al. ${ }^{(32)}$, podem alterar a composição da biota natural. 
As densidades do plâncton variaram entre os sistemas de cultivo e a biomassa de estocagem dos camarões e peixes, pois estas estão diretamente relacionadas com o aporte de nutrientes que entra no sistema e influencia a dinâmica do plâncton ${ }^{(33)}$. Melo et al. ${ }^{(34)}$ avaliaram três sistemas de cultivo de L. vannamei (orgânico, semi-intensivo e intensivo) e observaram diferenças na densidade do fitoplâncton. Casé et al. ${ }^{(35)}$ também observaram aumento na densidade do fitoplâncton com aumento da densidade de camarão.

Para o sistema de policultivo camarão-tilapia, Yuan et al. ${ }^{(13)}$ observaram alterações nos níveis de clorofila-a com o aumento da densidade de tilápia. Resultados similares foram observados por Muangkeow et al. ${ }^{(33)}$, quando avaliaram diferentes biomassas de estocagem de tilápia no policultivo com camarões marinhos.

A tilápia está entre as poucas espécies de peixes da aquicultura que se alimentam de alimentos naturais de baixo nível trófico, tais como detritos e plâncton ${ }^{(8)}$, e parece ser a escolha mais adequada para um policultivo camarão-peixe ${ }^{(36)}$. Entretanto, não ocorreram reduções significativas na densidade do plâncton com o aumento da biomassa de camarões e tilápias no presente estudo. Estes resultados diferem dos dados observados por Muangkeow et al. ${ }^{(33)}$, em que o aumento da densidade de estocagem da segunda espécie ocasionou aumento da densidade do fitoplâncton, principalmente de menor tamanho $(<5 \mu \mathrm{m})$. Esta diferença provavelmente esta relacionada às diferenças de densidade de peixes e camarões adotadas nos dois estudos e às quantidades de rações ofertadas.

Em monocultivo de camarões marinhos ocorre predominância de Cyanophyta na composição do fitoplâncton ${ }^{(20,21,37)}$, que, segundo Jú et al. ${ }^{(38)}$, são indesejáveis, pois são causadoras da perda da qualidade da água por reduzirem a transparência e os níveis de oxigênio na água e no sedimento, além de resultar em mortalidades ${ }^{(39,40)}$ e redução no crescimento dos camarões ${ }^{(37)}$. Somando-se a isto, tilápia é uma das poucas espécies de peixes que não são capazes de digerir Cyanophyta ${ }^{(36)}$. Já no monocultivo de tilápias em viveiros ocorre predominância de Chlorophyta ${ }^{(41)}$. Sun et al. ${ }^{(36)}$ observaram predominância de Cyanophyta em policultivo de camarões marinhos e tilápia e Uddin et al. ${ }^{(42)}$ de Chlorophytas em policultivo de camarões de água doce e tilápia. Esse resultados são diferentes dos observados no presente estudo, em que ocorreu a predominância de Bacillariophyta. A maior abundância de Bacillariophyta provavelmente está relacionada à salinidade da água e ao manejo de fertilização com nitrato de sódio, pois Boyd ${ }^{(43)}$ relata que este tipo de fertilizante é mais eficiente no desenvolvimento deste grupo. Segundo Jú et al. ${ }^{(38)}$, Bacillariophyta são os representantes do fitoplâncton que mais favorecem o crescimento dos camarões, além de indicarem uma boa produtividade natural nos ambientes de cultivo.

Em relação à comunidade zooplanctônica, em cultivo de camarões marinhos ocorre predominância de Copépoda na composição do zooplâncton ${ }^{(44)}$, que segundo Martínez-Córdova et al. ${ }^{(45)}$ são desejáveis, pois melhoraram a sobrevivência e o fator de conversão alimentar de juvenis de camarões marinhos, provavelmente pela boa composição centesimal de lipídios e proteínas de alta qualidade $^{(46)}$. Já para monocultivo de tilápia em viveiros ocorre predominância de Rotífera (zooplâncton) ${ }^{(41)}$.

Sun et al. ${ }^{(36)}$ observaram predominância de Copépoda em policultivo de camarões marinhos e tilápia. Esse resultado difere dos observados neste estudo, no qual ocorreu a predominância de Rotífera. Provavelmente tal fato está relacionado com a adaptação destes organismos para níveis mais elevados de nutrientes e sólidos, pois em sistema sem renovação de água há predominância deste grupo $^{(26,35)}$. 
As densidades de Bacillariophyta, Cyanophyta e Chlorophyta estiveram respectivamente dentro das

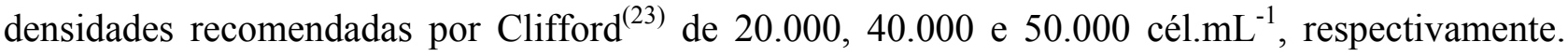
Entretanto, as densidades de Pyrrophyta estavam acima do recomendado de 500 cél. $\mathrm{mL}^{-1}$ (Tabela 2). O aumento na densidade de Pyrrophyta pode ser um problema durante o período de cultivo, pois este grupo é considerado como uma das causas do retrocesso da maricultura Chinesa, por causa da floração de algumas espécies nocivas ${ }^{(47)}$. Já as densidades de Rotífera e Copépoda estiveram, respectivamente, abaixo das densidades recomendadas por Clifford ${ }^{(24)}$ de $2.000-50.000$ org. $L^{-1}$ (Tabela 2).

A diferença na composição do plâncton entre os trabalhos citados está relacionada à dinâmica dos sistemas de cultivo (fertilizações, alimentação, densidades de estocagem, fontes de abastecimento de água), enquanto as diferenças nas variáveis ambientais (salinidade, temperatura, oxigênio dissolvido, amônia, demanda bioquímica do oxigênio) estão relacionadas com a enorme diversidade de grupos e espécies.

Independentemente da criação principal ser a tilápia ou o camarão, não foi observada redução na densidade da biota natural nas unidades experimentais com policultivo à medida que a densidade da segunda espécie aumentou; no entanto, foi observada predominância de Bacillariophyta e Rotífera.

\section{Conclusões}

Em sistema de policultivo do camarão marinho branco do Pacífico e tilápia do Nilo em água com baixa salinidade e sem renovação, não ocorrem modificações na composição da biota natural à medida que se aumenta a biomassa da segunda espécie cultivada, sendo Bacillariophyta e Rotífera os principais representantes da composição do fitoplâncton e zooplâncton, respectivamente.

\section{Agradecimentos}

Os autores agradecem ao professor Dr. Alfredo Olivera Gálvez do Departamento de Pesca e Aquicultura da Universidade Federal Rural de Pernambuco pela ajuda e disponibilidade do Laboratório de Produção de Alimento Vivo (LAPAVI) para realização das análises do plâncton.

\section{Referências}

1. Brito LO, Dantas DMM, Pereira-Neto JB, Soares R, Olivera A. Efeito de duas estratégias de fertilização na composição do fitoplâncton no cultivo de Litopenaeus vannamei. Arquivos de Ciência do Mar. [periódico na Internet]. 2009; 42(1): 30-35. Disponível em: http://www.periodicos.ufc.br/index.php/arquivosdecienciadomar/article/view/6036/4251

2. Brito LO, Pimentel LF, Marcelino SC, Melo VF, Costa WM, Olivera A. Efeito de duas estratégias de fertilização na composição do zooplâncton no cultivo de Litopenaeus vannamei. Revista Brasileira Engenharia de Pesca. [periódico na Internet]. 2009; 4(1): 57-66. Disponível em: http://ppg.revistas.uema.br/index.php/REPESCA/article/view/130/120 
3. Brito LO, Pereira-Neto JB, Dantas DMM, Vasconcelos RFL, Gálvez AO. Efeito de diferentes fertilizantes inorgânicos na composição do fitobentos em viveiros de cultivo de Litopenaeus vannamei. Arquivos de Ciências do Mar. [periódico na Internet]. 2014, 47(1), 5 - 14. Disponível em: http://www.periodicos.ufc.br/index.php/arquivosdecienciadomar/article/view/5953/4389

4. Martínez-Córdova LR, Peña-Messina E. Biotic communities and feeding habits of Litopenaeus vannamei (Boone 1931) and Litopenaeus stylirostris (Stimpson 1974) in monoculture and polyculture semi-intensive ponds. Aquaculture Research. [periódico na Internet]. 2005, 36: 1075-1084. Disponível em: http:/onlinelibrary.wiley.com/doi/10.1111/j.1365-2109.2005.01323.x/full

5. Silva CF, Ballester E, Monserrat J, Geracitano L, Wasielesky W, Abreu PC. Contribution of microorganisms to the biofilm nutritional quality: protein and lipid contents. Aquaculture Nutrition. [periódico na Internet]. 2008; 14: 507-514. Disponível em: http:/onlinelibrary.wiley.com/doi/10.1111/j.1365-2095.2007.00556.x/full

6. Zhou Q, Li K, Jun X, Bo L. Role and functions of beneficial microorganisms in sustainable aquaculture. Bioresource Technology. [periódico na Internet]. 2009, 100: 8780-3786. Disponível em: http://www.sciencedirect.com/science/article/pii/S0960852408010973

7. Dias ACMI, Branco CWC, Lopes VG. Estudo da dieta natural de peixes no reservatório de Ribeirão das Lajes, Rio de Janeiro, Brasil. Acta Scientiarum Biological Sciences. [periódico na Internet]. 2005, 27 (4): 355-364. Disponível em: http://eduem.uem.br/ojs/index.php/ActaSciBiolSci/article/view/1270/730

8. Vicente IST, Fonseca-Alves CE. Impact of introduced nile tilapia (Oreochromis niloticus) on non-native aquatic ecosystems. Pakistan Journal of Biological Sciences. [periódico na Internet]. 2013, 16 (3): 121-126. Disponível em: http://dx.doi.org/10.3923/pjbs.2013.121.126.

9. Bosma RH, Verdegem MCJ. Sustainable aquaculture in ponds: principles, practices and limits. Livestock Science. [periódico na Internet]. 2011, 139: 58-68. Disponível em: http://www.sciencedirect.com/science/article/pii/S1871141311001004

10. Saraswathy R, Muralidhar M, Ravichandran P, Ponniah AG, Panigrahi A, Kailasam M, Nagavel A. Effect of nutrient level on phytoplankton population in zero water exchange shrimp culture farms. Indian Journal Fish. [periódico na Internet]. 2012, 59 (2): 115-120. Disponível em: http://epubs.icar.org.in/ejournal/index.php/IJF/article/view/11414/10482.

11. Barbieri RCJ, Ostrensky AN. Camarões marinhos - reprodução, maturação e larvicultura. 1st ed. Viçosa: Aprenda Fácil, 2002, 255p.

12. Beyruth Z, Mainardes-Pinto CSR, Fusco SM, Faria FC, Silva AL. Utilização de alimentos naturais por Oreochromis niloticus em tanques de terra com arraçoamento. Boletim do Instituto de Pesca. [periódico na Internet]. 2004, 30 (1): 9 - 24. Disponível em: ftp://ftp.sp.gov.br/ftppesca/Beyruth30 1.pdf

13. Yuan D, Yi Y, Yakupitiyage A, Fizimmons K, Diana JS. Effects of addition of red tilapia (Oreochromis spp.) at different densities and sizes on production water quality and nutrient recovery of intensive culture of white shrimp (Litopenaeus vannamei) in cement tanks. Aquaculture. [periódico na Internet]. 2010, 298: 226238. Disponível em: http://www.sciencedirect.com/science/article/pii/S0044848609009272

14. Simão BR, Brito LO, Maia ASC, Miranda LC, Azevedo CMDSB. Stocking densities and feeding strategies in shrimp and tilapia polyculture in tanks. Pesquisa Agropecuária Brasileira. [periódico na Internet]. 2013， 48: 1088-1095. Disponível em: http://www.scielo.br/scielo.php?pid=S0100204X2013000800042\&script $=$ sci arttext

15. Wang M, Lu M. Tilapia polyculture: a global review. Aquaculture Research. [periódico na Internet]. 2016, 47 (8): 2363-2374. Disponível em: http://onlinelibrary.wiley.com/doi/10.1111/are.12708/full 
16. Danaher JJ, Tidwell JH, Coyle SD, Dasgupta S, Zimba PVA. Effects of two densities of caged monosex nile tilapia, Oreochromis niloticus, on water quality, phytoplankton populations, and production when polycultured with Macrobrachium rosenbergii in temperate ponds. Journal of the World Aquaculture Society. [periódico na Internet]. 2007. 38 (3): 367-382. Disponível em: http://onlinelibrary.wiley.com/doi/10.1111/j.1749-7345.2007.00109.x/full

17. Campos AAB, Maia EP, Costa WM, Brito LO, Oliveira A. O. Qualidade da água em fazenda de camarão marinho Litopenaeus vannamei com sistema de recirculação parcial. Ciência Animal Brasileira. [periódico na Internet]. 2008: 9 (4): 819-826. Disponível em: https://www.revistas.ufg.br/vet/article/view/522

18. Messias GA, Brito LO, Maia EP, Souza FMMC, Costa WM, Oliveira A. Caracterização da qualidade da água em fazenda de carcinicultura marinha, com sistema fechado de recirculação. Pesquisa Agropecuária Pernambucana. 2010: 15: 35-41.

19. Maia EP, Olivera A, Brito LO. Brazilian shrimp farms for Litopenaeus vannamei with partial and total recirculation systems. International Journal Aquaculture Science. [periódico na Internet].2011: 2 (1): 16-27. Disponível em: http://www.journal-aquaticscience.com/2011(1)/Maia\%20et\%20al\%20(2011).pdf

20. Campos AAB, Maia EP, Costa WM, Brito LO, Olivera AO. Descrição dos principais grupos fitoplânctonicos do afluente e efluente em fazenda de criação do camarão marinho Litopenaeus. vannamei com sistema de recirculação parcial de água. Boletim do Instituto de Pesca. [periódico na Internet]. 2007. 33 (1):113 - 119. Disponível em: http://www.pesca.sp.gov.br/33_1_113-119.pdf

21. Messias GA, Brito LO, Maia EP, Souza FMMC, Costa WM, Olivera A. Caracterização do fitoplâncton em fazenda de carcinicultura marinha, com sistema fechado de recirculação. Pesquisa Agropecuária Pernambucana. 2010. 15: 29-34.

22. Paiva-Maia E, Alves-Modesto G, Otavio-Brito L, Olivera A, Vasconcelos-gesteira TC. Effect of a commercial probiotic on bacterial and phytoplankton concentration in intensive shrimp farming (Litopenaeus vannamei) recirculation systems. Latin American Journal Aquatic Research. [periódico na Internet]. 2013. 41

(1): 126-137. Disponível em: http://www.scielo.cl/scielo.php?pid=S0718560X2013000100010\&script=sci arttext\&tlng=en

23. Clifford HC. Semi-intensive sensation: a case study in marine shrimp pond management. World Aquaculture. 1994; 25 (6): 98-102.

24. Kubitza F. Tecnologia e planejamento na produção comercial de tilápias. 1st ed. Jundiaí: Aqua Imagem, 2000, 285p. Portuguese

25. Pereira-Neto JB, Dantas DMM, Gálvez AO, Brito LO. Avaliação das comunidades planctônica e bentônica de microalgas em viveiros de camarão (Litopenaeus vannamei). Boletim do Instituto de Pesca. [periódico na Internet]. 2008. 34 (4): 543-551. Disponível em: ftp://ftp.sp.gov.br/ftppesca/34 4 543-551.pdf

26. Ferreira-Marinho Y, Brito LO, Silva CVF, Santos IGS, Gálvez AO. Effect of addition of Navicula sp. on plankton composition and postlarvae growth of Litopenaeus vannamei reared in culture tanks with zero water Exchange. Latin American Journal Aquatic Research. [periódico na Internet]. 2014. 42 (3): 427-437. Disponível em: http://www.scielo.cl/scielo.php?pid=S0718-560X2014000300004\&script=sci arttext

27. Stanfford C. A guide to Phytoplankton of Aquaculture Ponds. 1st ed. Queensland: Collection analysis and Identification, 1999, 59p.

28. Sipaúba-Tavares LH, Rocha O. Produção de plâncton (zooplâncton e fitoplâncton) para alimentação de organismos aquáticos. 1st ed. São Carlos: RIMA, 2001, 106p.

29. Alves CS, Mello GL. Manual prático de monitoramento de qualidade de água e solo em aqüicultura. 1st ed. Recife: FAEPE, 2007, 58p. 
30. Kubitza F. Qualidade da água no cultivo de peixes e camarões. 1st ed. Jundiaí: Aqua Imagem, 2003, 229 p.

31. Ferreira NC, Bonetti C, Seiffert WQ. Hydrological and water quality indices as management tools in marine shrimp culture. Aquaculture. [periódico na Internet]. 2011. 318: 425-433. Disponível em: http://www.sciencedirect.com/science/article/pii/S0044848611004509

32. Preston NP, Coman FE, Fry VM. Shrimp pond zooplankton dynamics and the efficiency of sampling effort. Aquaculture Research. [periódico na Internet]. 2003. 34: 373-381. Disponível em: http://onlinelibrary.wiley.com/doi/10.1046/j.1365-2109.2003.00797.x/abstract

33. Muangkeow B, Ikegima K, Powtongook S, Yi Y. Effects of white shrimp, Litopenaeus vannamei (Boone) and Nile tilapia, Oreochromis niloticus L., stocking density on growth, nutrient conversion rate and economic return in integrated closed recirculation system. Aquaculture. [periódico na Internet]. 2007. 269: 363-376. Disponível em: http://www.sciencedirect.com/science/article/pii/S0044848607002980

34. Melo MP, Carvalheiro JM, Cordeiro TA, Queiroz AR, Prado JP, Borges IF. Phytoplanktonic composition of three cultivation systems used in Litopenaeus vannamei (Boone, 1931) marine shrimp farms. Acta Scientiarum Biological Sciences. [periódico na Internet]. 2010. 32 (3): 223-228. Disponível em: http://ojs.uem.br/ojs/index.php/ActaSciBiolSci/article/view/4816/4816

35. Casé M, Leça EE, Leitão SN, Santanna EE, Schwamborn R, Junior ATM. Plankton community as indicator of water quality in tropical shrimp culture ponds. Marine Pollution Bulletin. [periódico na Internet]. 2008. 56: 1343-1352. Disponível em: http://www.sciencedirect.com/science/article/pii/S0025326X08000805

36. Sun W, Dong S, Jie Z, Zhao Z, Zhang H, Li J. The impact of net-isolated polyculture of tilapia (Oreochromis niloticus) on plankton community in saline-alkaline pond of shrimp (Penaeus vannamei). Aquaculture International. [periódico na Internet]. 2011. 19: 779-788. Disponível em: https://link.springer.com/article/10.1007/s10499-010-9394-8

37. Alonso-Rodríguez R, Páez-Osuna F. Nutrients, phytoplankton and harmful algal blooms in shrimp ponds: a review with special reference to the situation in the Gulf of California. Aquaculture. [periódico na Internet]. 2003. 219: 317-336. Disponível http://www.sciencedirect.com/science/article/pii/S0044848602005094

38. Jú ZY, Forster L, Conquest L, Dominy W, Kuo WC, Horgen FD. Determination of microbial community structures of shrimp floc cultures by biomarkers and analysis of floc amino acid profiles. Aquaculture Research. [periódico na Internet]. 2008. 39: 118-133. Disponível em: http://onlinelibrary.wiley.com/doi/10.1111/j.1365-2109.2007.01856.x/full

39 Pérez-Linares J, Cadena M, Rangel C, Venzueta-Bustamante ML, Ocha JL. Effect of Schizothrix calcicola on white shrimp Litopenaeus vannamei (Penaeus vannamei) postlarvae. Aquaculture. [periódico na Internet]. 2003. 218: 55-65. Disponível http://www.sciencedirect.com/science/article/pii/S0044848602004477

40. Zimba PV, Camus A, Allen EH, Burkholder JM. Co-occurrence of white shrimp, Litopenaeus vannamei, mortalities and microcystin toxin in a southeastern USA shrimp facility. Aquaculture. [periódico na Internet]. 2006. 261: 1048-1055. Disponível em: http://www.sciencedirect.com/science/article/pii/S0044848606006648

41. Moreira RF, Silveira LP, Teixeira EG, Moreira AGL, Moura PS, Frias WRL. Growth and gastrointestinal indices in Nile tilapia fed with different Diets. Acta Scientiarum Animal Science [periódico na Internet]. 2012. 34 (3): 223-229. Disponível em: http://www.scielo.br/scielo.php?pid=S1807$\underline{86722012000300002 \& \text { script }=\text { sci arttext }}$ 
42. Uddin MS, Rahman SMS, Azim ME,Wahab MA, Verdegem MCJ, Verreth JAJ. Effects of stocking density on production and economics of Nile tilapia (Oreochromis niloticus) and freshwater prawn (Macrobrachium rosenbergii) polyculture in periphyton-based systems. Aquaculture Research. [periódico na Internet]. 2007. 38: 1759-1769. Disponível em: http://onlinelibrary.wiley.com/doi/10.1111/j.13652109.2007.01837.x/full

43. Boyd CE. Pond bottom soil and water quality management for pond aquaculture. 1st ed. Alabama: ASA. 1997. 55p. Inglês,

44. Porchas-Cornejo MA, Martínez-Córdova LR, Martínez-Porchas M, Barraza-Guardado R, RamosTrujillo, L. Study of zooplankton communities in shrimp earthen ponds, with and without organic nutrientenriched substrates. Aquaculture International. [periódico na Internet]. 2013. 21: 65-73. Disponível em: https://ink.springer.com/article/10.1007/s10499-012-9534-4

45. Martínez-Córdova LR, Campaña-Torres A, Martínez-Porchas M. Evaluación del suministro de cuatro densidades de copépodos (Acartia sp y Calanus pacificus) sobre la respuesta productiva de Litopenaeus vannamei (Boone 1931) preengordado intensivamente a nivel microcosmos. Ciencias Marina. [periódico na Internet]. 2011. 37: 415-423. Disponível em: http://www.scielo.org.mx/scielo.php?script=sci arttext\&pid=S0185-38802011000400004

46. Martínez-Córdova LR, Campaña-Torres A, Martínez-Porchas M. Manejo de la Productividad Natural en el Cultivo del Camarón. In: Cruz-Suárez LE, Ricque-Marie D, Nieto-López MG, Villarreal D, Scholz U, González M. Avances em Nutrición Acuícola. Sonora: Memorias del VII Simposium Internacional de Nutrición Acuícola, 2004, p.671-694.

47. Yan T, Zitou M, Fu M, Yu R, Wang Y, Ci J. Effects of the dinoflagellate Alexandrium tamarense on early development of the scallop Argopecten irradians concentricus. Aquaculture. [periódico na Internet]. 2003. 277: 167-178. Disponível em: http://www.sciencedirect.com/science/article/pii/S0044848602001175 